\title{
Interleukin 10 in Helicobacter pylori associated gastritis: immunohistochemical localisation and in vitro effects on cytokine secretion
}

\author{
K Bodger, K Bromelow, J I Wyatt, R V Heatley
}

\begin{abstract}
BackgroundIAims-Interleukin 10 (IL-10) is a counterinflammatory peptide implicated in the downregulation of human intestinal immune responses. Enhanced secretion of IL-10 has been documented in gastric biopsy organ culture in Helicobacter pylori infection. This study aimed to define the cellular origins of IL-10 in $H$ pylori associated gastritis, and to determine the effects of endogenous IL-10 on proinflammatory cytokine secretion in vitro.
\end{abstract}

Methods-Endoscopic biopsies were obtained from the gastric antrum at endoscopy from patients with dyspepsia. Two pairs of antral biopsies were cultured in vitro for $\mathbf{2 4}$ hours, one pair in the presence of neutralising anti-IL-10 monoclonal antibody, the other pair as controls. The cytokine content of culture supernatants (tumour necrosis factor $\alpha$ (TNF- $\alpha$ ), IL-6, and IL-8) was determined by enzyme linked immunosorbent assay and corrected for biopsy weight. Helicobacter pylori status was established by histology and biopsy urease test, and histopathology graded by the Sydney system. In a subgroup of patients, western blotting was used to establish CagA serological status. Immunohistochemistry for IL-10 was performed on formalin fixed tissues using a combination of microwave antigen retrieval and the indirect avidin-biotin technique. Immunoreactivity was scored semiquantitatively.

Results-In vitro culture was performed in 41 patients: 31 with $H$ pylori positive chronic gastritis and $10 \mathrm{H}$ pylori negative. In vitro secretion of TNF- $\alpha$, IL-6, and IL-8 for "control" biopsies was significantly higher in $\boldsymbol{H}$ pylori positive versus negative samples, with values of TNF- $\alpha$ and IL-6 correlating with the degree of active and chronic inflammation and being higher in CagA seropositive cases. No evidence for enhanced cytokine secretion was seen in biopsies cocultured in the presence of anti-IL-10 monoclonal antibody. Immunohistochemistry was performed in 29 patients, of whom 13 were $H$ pylori positive. IL-10 immunoreactivity was observed in the surface epithelium in all $H$ pylori positive cases and in 13 of 16 negative cases, especially in areas of surface epithelial degeneration. Lamina propria mononuclear cells (LPMNCs) were positively stained in all $H$ pylori positive cases and in 12 of 16 negative cases, with a significantly greater proportion of positive LPMNCs in the positive group.

Conclusions-This study localised IL-10 protein to the gastric epithelium and LPMNCs. In vitro proinflammatory cytokine secretion was increased in $\mathrm{H}$ pylori infection (especially CagA positive infection), but blocking endogenous IL-10 secretion did not significantly increase cytokine secretion. IL-10 is implicated in $H$ pylori infection and might "damp down" local inflammation. The role of gastric IL-10 secretion in determining the clinicopathological outcome of infection merits further study.

(f Clin Pathol 2001;54:285-292)

Keywords: Helicobacter pylori infection; interleukin 10; gastritis; immunohistochemistry

Despite the apparent activation of host cellular and humoral immune responses in Helicobacter pylori infection, colonisation of the gastric mucosa is generally persistent and results in chronic inflammation. ${ }^{1}$ The severity of $H$ pylori associated chronic gastritis and the clinical outcome are highly variable between individuals, with only a minority developing serious gastroduodenal disease. The factors that contribute to failure of the immune response to clear the organism and to the varied clinicopathological picture are poorly understood. The outcome of infection is likely to reflect a complex interaction between constitutional host factors, variation in virulence between infecting $H$ pylori strains (for example, strains possessing the cagA pathogenicity island of genes), ${ }^{2}$ and environmental cofactors. ${ }^{1}$

Cytokines are responsible for orchestrating the immune response, and we and others have reported the increased secretion of proinflammatory peptides (such as tumour necrosis factor $\alpha$ (TNF- $\alpha$ ), interleukin 1 (IL-1) $\alpha / \beta$, IL-6, and IL-8) in $H$ pylori infection..$^{3-9}$ Less attention has been focused on the natural antagonists of these stimulatory cytokines. IL-10 is a potent anti-inflammatory cytokine with a variety of biological effects, including inhibition of many of the effector functions of phagocytes, such as the synthesis of proinflammatory cytokines (for example, TNF- $\alpha$, IL-6, and IL-8). ${ }^{10}$ The chronic enterocolitis developing in IL-10 (-/-) knockout mice suggests a role for this peptide in maintaining the normal noninflamed state of the intestinal mucosa despite 
continuous antigen exposure. ${ }^{11}$ We postulated that IL-10 might contribute towards the failure of the immune response to clear $H$ pylori infection, and in a previous study we found increased secretion of IL-10 in biopsy organ culture in $H$ pylori infection, with secretion of the cytokine correlating with the degree of chronic inflammation. ${ }^{3}$ Yamaoka et al have reported increased expression of IL-10 mRNA in biopsies from infected patients. ${ }^{79} \mathrm{~A}$ role for IL-10 in "damping down" the immune response to helicobacter infection is supported by the recent observation of the rapid development of a severe hyperplastic gastritis in Helicobacter felis infected IL-10 (-/-) knockout mice. ${ }^{12}$ The cellular sources of IL-10 and its effects on cytokine secretion in chronic $H p y$ lori infection are unknown.

In our present study, we aimed to: (1) define further the effects of IL-10 on proinflammatory cytokine secretion in $H$ pylori infection; (2) correlate cytokine responses with histopathological features of gastritis and with CagA serological status; and (3) determine the cellular sources of IL-10 in the gastric antrum by means of immunohistochemistry.

\section{Methods}

PATIENTS AND BIOPSY SAMPLES FOR IN VITRO CULTURE

Consecutive patients with dyspepsia attending for diagnostic endoscopy were recruited, after obtaining informed consent. Patients who had received (within the last month) antibiotics, proton pump inhibitors, bismuth containing compounds, non-steroidal anti-inflammatory drugs, corticosteroids, or immunosuppressive drugs were excluded, as were patients with a known history of malignancy or immunosuppression. Our study was approved by the Leeds healthcare clinical research (ethics) committee.

During upper gastrointestinal endoscopy, multiple biopsies were taken from adjacent sites of the gastric antrum (within $3 \mathrm{~cm}$ of the pylorus), avoiding areas of macroscopic erosion or ulceration. Routine targeted biopsies were taken for diagnostic purposes using a standard procedure. Standard "pinch" biopsy forceps were used. Six antral biopsies were taken from each patient. Four biopsies were subject to in vitro culture (in pairs), one biopsy was fixed in $10 \%$ buffered formalin for histopathological processing, and one biopsy was used for biopsy urease testing (CLO test; Delta West Ltd, Perth, Western Australia). In addition, a $10 \mathrm{ml}$ sample of venous blood was taken and serum was separated by centrifugation and stored at $-20^{\circ} \mathrm{C}$ until subsequent western blot assay in a subgroup of patients.

HISTOPATHOLOGY

Serial $5 \mu \mathrm{m}$ sections of formalin fixed, paraffin wax embedded sections were stained by both haematoxylin and eosin and modified Giemsa according to histopathological standards for light microscopy. Histology of routinely stained sections was subsequently reported by an experienced histopathologist (JIW) using the updated Sydney system, ${ }^{13}$ blinded for the results of the cytokine estimations. A grade from zero (absent) to 3 (severe) was assigned for five histological parameters; namely, inflammation (chronic inflammatory cells), activity (neutrophils), glandular atrophy, intestinal metaplasia, and $H$ pylori density.

\section{DETERMINATION OF H PYLORI STATUS}

Giemsa staining was used to identify $H$ pylori in paraffin wax sections of formalin fixed antral biopsies, and the result of the biopsy urease test was read within 24 hours. The criteria for $H$ pylori status were: negative, all tests negative; positive, at least one test positive.

\section{IN VITRO ORGAN CULTURE}

Culture medium comprised RPMI-1640 medium, supplemented with $10 \%$ fetal calf serum and antibiotics. ${ }^{3}$ In the endoscopy suite, biopsies for in vitro culture were immediately placed in culture medium at $4^{\circ} \mathrm{C}$ and transported on ice to the laboratory. Biopsies were then placed in pairs in tissue culture wells containing $0.5 \mathrm{ml}$ fresh culture medium as described previously. ${ }^{31415}$ One pair of biopsies from each patient was cultured in culture medium alone ("control" pair), whereas the remaining pair of biopsies was cultured in medium containing $1 \mu \mathrm{g} / \mathrm{ml}$ of mouse antihuman IL-10 neutralising monoclonal antibody (R\&D Systems, Minneapolis, Minnesota, USA). This concentration was determined from the anticipated levels of endogenous IL-10 secretion (according to our previous work $)^{3}$ and reference to the manufacturer's data on the neutralisation capacity of the antibody, ensuring an excess of neutralising anti-IL-10 antibody.

Biopsies were then cultured for 24 hours at $37^{\circ} \mathrm{C}$ in a $5 \% \mathrm{CO}_{2}$ humidified incubator. At 24 hours, the culture medium was removed and the cells were centrifuged at $2000 \times g$ for 15 minutes at $4^{\circ} \mathrm{C}$, snap frozen, and stored at $-80^{\circ} \mathrm{C}$ until cytokine analysis. After culture, biopsies were dried to remove secreted mucus by gently turning on fresh, sterile filter papers and the wet weight determined as described previously. ${ }^{3}$

\section{CYTOKINE ESTIMATIONS BY ENZYME LINKED} IMMUNOASSAY

The cytokine content of culture supernatants was determined with the use of commercial microtitre plate enzyme linked immunosorbent assays (ELISAs), according to the manufacturers' instructions. All assays were based on a quantitative sandwich enzyme immunoassay technique. To allow multiple cytokine estimations, culture supernatants were prediluted in phosphate buffered saline (PBS; $1 / 4$ to $1 / 64$ depending on assay) before measuring the cytokine content, and values were adjusted for the dilution factor as appropriate. All assays were performed in duplicate. Cytokine secretion was normalised for biopsy wet weight and expressed as $\mathrm{pg} / \mathrm{mg} / 24$ hours.

The ELISA assays all used a microtitre plate precoated with a monoclonal antibody directed against the cytokine to be assayed, to which samples and standards were added and plates were incubated. After washing, an enzyme 
Table 1 Patient characteristics, histopathology (Sydney) scores, and cytokine secretion ("control" biopsies)

\begin{tabular}{lll}
\hline & Helicobacter pylori + ve & Helicobacter pylori - ve \\
\hline Number & 31 & 10 \\
Mean age (range) & $57.9(32-84)$ & $57.4(23-87)$ \\
Male/female ratio & $18 / 13$ & $5 / 5$ \\
Antral inflammation & & \\
Absent or mild $(0,1)$ & $12 / 31$ & $10 / 10$ \\
Moderate or severe $(2,3)$ & $19 / 31$ & $0 / 10$ \\
Antral activity & & $10 / 10$ \\
Absent or mild $(0,1)$ & $20 / 31$ & $0 / 10$ \\
Moderate or severe $(2,3)$ & $11 / 31$ & \\
Cytokine secretion & & $0.00(0.00-0.18)$ \\
TNF- $\alpha$ & $0.72^{\star}(0.27-1.20)$ & $10.50(5.65-12.72)$ \\
Interleukin 6 & $27.30^{\star}(14.30-67.30)$ & $324(269.5-533.7)$ \\
Interleukin 8 & $1122^{\star}(684-1864)$ & \\
\hline
\end{tabular}

Values for cytokine secretion are median (interquartile range), expressed as $\mathrm{pg} / \mathrm{mg} / 24$ hours. ${ }^{\star} \mathrm{p}<0.05$ for $H$ pylori $+\mathrm{ve} v$ H pylori - ve (Mann Whitney U test).

TNF- $\alpha$, tumour necrosis factor $\alpha$.

linked polyclonal antibody specific for the cytokine was added and the plate was incubated and then washed again. A substrate solution was then added and after stopping the reaction the colour intensity was read by automated spectrophotometer. TNF- $\alpha$ was measured by means of a high sensitivity ELISA that uses an additional amplification step (HS "Quantikine"; R\&D Systems), whereas IL-6 (Biotrak ELISA; Amersham Life Science, Amersham, Buckinghamshire, UK) and IL-8 ("Quantikine" ELISA; R\&D Systems) were assayed by standard ELISA as described above. According to the manufacturers' data, the sensitivity of these assays were: TNF- $\alpha,<0.11 \mathrm{pg} /$ $\mathrm{ml}$; IL-6, $<1 \mathrm{pg} / \mathrm{ml}$; IL-8, < $10 \mathrm{pg} / \mathrm{ml}$. No crossreactivity has been reported for other cytokines.

\section{IMMUNOHISTOCHEMISTRY}

Biopsy material comprised archival formalin fixed, paraffin wax embedded endoscopic antral biopsies from 29 cases of known $H$ pylori status (by histology and urease testing), obtained from the pathology department, St James's University Hospital. Serial sections were subject to indirect immunohistochemistry using a combination of microwave antigen retrieval $^{16}$ and a standard indirect sequential avidin-biotin technique with 3,3'diaminobenzidine tetrahydrochloride (DAB) development. ${ }^{17}$

Sections were dewaxed in xylene, rehydrated with graded alcohol, and washed in water. Endogenous peroxidase activity was blocked in $2 \%$ hydrogen peroxide in methanol and slides were washed. Pilot experiments established that specific staining was optimal using microwave antigen retrieval, ${ }^{16}{ }^{17}$ rather than untreated paraffin wax sections. Sections were subject to two incubation cycles of five minutes in plastic coplin jars containing citrate buffer (10 nmol/1 citric acid, $\mathrm{pH} 6.0$ ), using a laboratory microwave oven with an intrinsic temperature monitoring probe inserted into the buffer solution, and the temperature limit set to $80^{\circ} \mathrm{C}$. Care was taken to ensure that biopsies were submerged in the buffer at all times. After cooling to room temperature for 20 minutes, sections were washed in PBS then incubated with rabbit serum buffer (1/20 in PBS) to block non-specific binding.
Sections were then incubated at room temperature in humidity chambers for two hours with optimal concentrations of primary antiserum (mouse antihuman IL-10 monoclonal antibody; R\&D Systems) diluted in serum buffer. Pilot experiments using a range of dilutions $(1 / 50$ to $1 / 3200)$ of stock primary antiserum $(0.5 \mathrm{mg} / \mathrm{ml})$ established an optimal concentration for anti-IL-10 immunostaining $(1 / 50)$. Normal serum buffer alone was used as a specificity control and all sections were negative when primary antiserum was omitted.

Slides were washed in running tap water, flooded with PBS, and drained. An aliquot of $50 \mu \mathrm{l}$ of biotinylated secondary antibody (rabbit antimouse) was added to each section and slides were incubated for 30 minutes. Slides were again washed and $50 \mu \mathrm{l}$ of peroxidase conjugated streptavidin was added to each slide and incubated for 30 minutes. The slides were washed again and developed for 10 minutes using fresh DAB solution. Sections were then counterstained with Mayer's haematoxylin for 30 seconds, washed in water, then dehydrated through alcohols. Finally, sections were cleared through xylene, mounted in DPX and subjected to light microscopy.

Immunostaining was graded semiquantitatively using a representative field viewed at high power. Separate scores were assigned for epithelium and lamina propria mononuclear cells (LPMNCs) as follows:

Epithelial staining: 0, absent; 1, weak; 2, moderate; 3, strong.

LPMNCs: (expressed as a proportion of positive cells) 0 , absent; 1 , scanty, isolated cells amounting to $<10 \%$ of total LPMNCs; 2, moderate numbers, $>10 \%$ but $<50 \%$ of total LPMNCs; 3, frequent, $>50 \%$ of LPMNCs.

DETERMINATION OF H PYLORI CagA STATUS

Sera from a subgroup of patients were subject to western blotting using a commercial kit for the identification of antibodies to the $120 \mathrm{kDa}$ CagA protein (RIDA Blot Helicobacter IgG; Quadratech, Epsom, Surrey) according to the manufacturer's instructions. The kit contains nitrocellulose strips containing $H$ pylori antigens that have previously been separated by weight using gel electrophoresis. Serum samples and positive and negative controls (supplied) are added to each strip in wells containing $2 \mathrm{ml}$ of blotting buffer. Sealed wells are then incubated on a horizontal shaker and subsequently washed repeatedly. Enzyme conjugated antihuman IgG is then added, and after incubation the strips washed again. IgG from the sample/control serum that has bound to the protein bands on the strip is then visualised using a substrate solution (diaminobenzidine).

\section{STATISTICAL ANALYSIS}

Cytokine secretion is expressed as $\mathrm{pg} / \mathrm{mg} / 24$ hours with median values and 25 th to 75 th interquartile ranges (IQR) given. Values for $H$ pylori positive and negative patients (control biopsies only) were compared using the MannWhitney U test. Paired values (controls and biopsies cocultured with the anti-IL-10 monoclonal antibody) within the $H$ pylori positive 
A

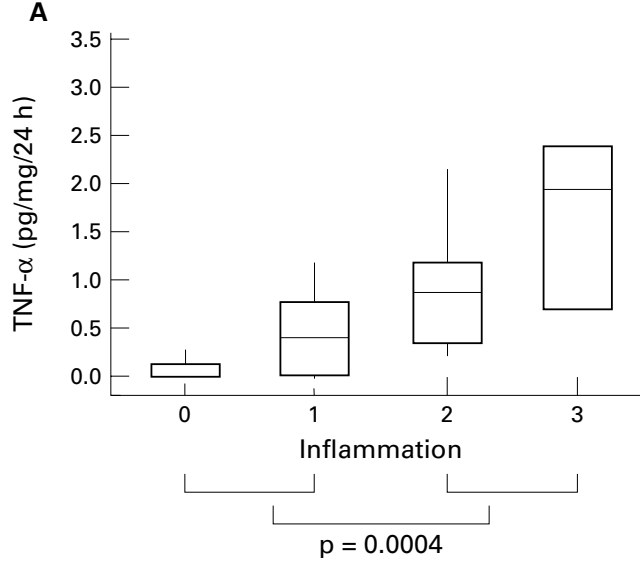

C

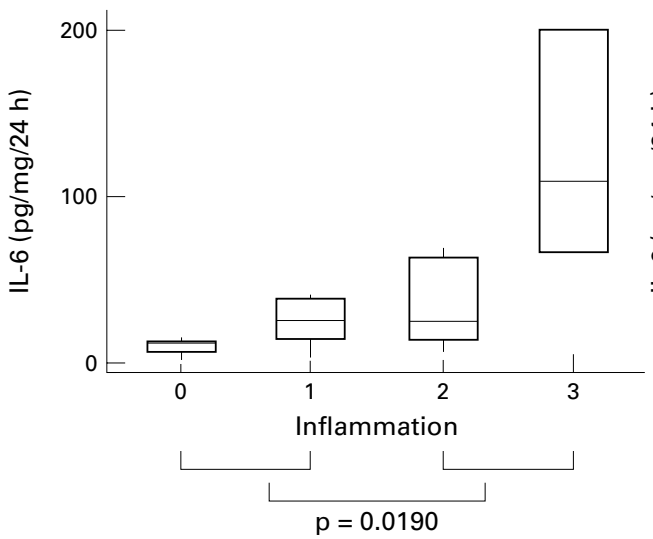

$\mathrm{E}$

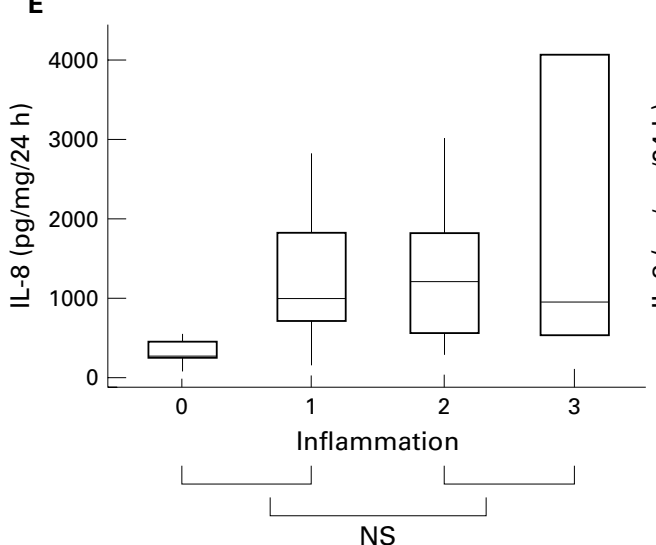

B

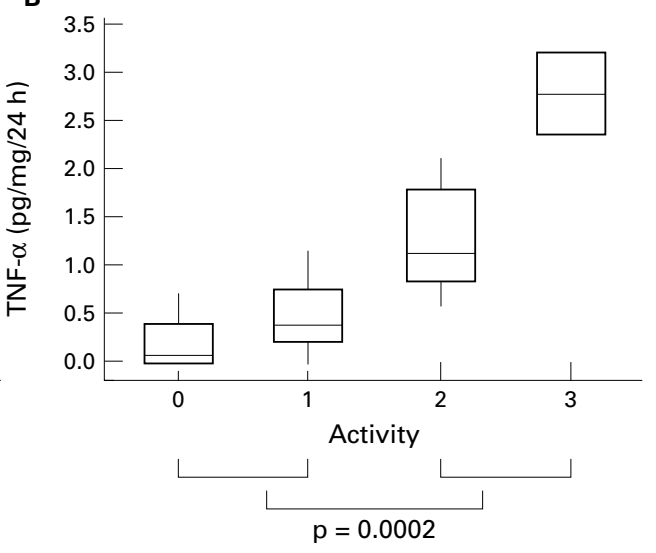

D

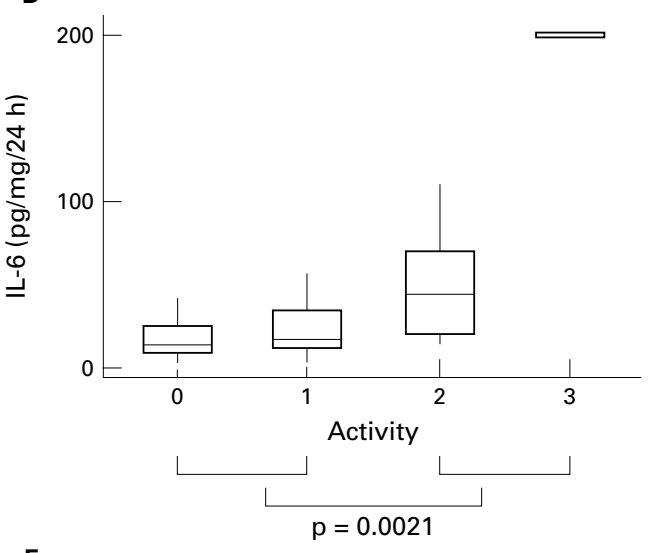

$\mathbf{F}$

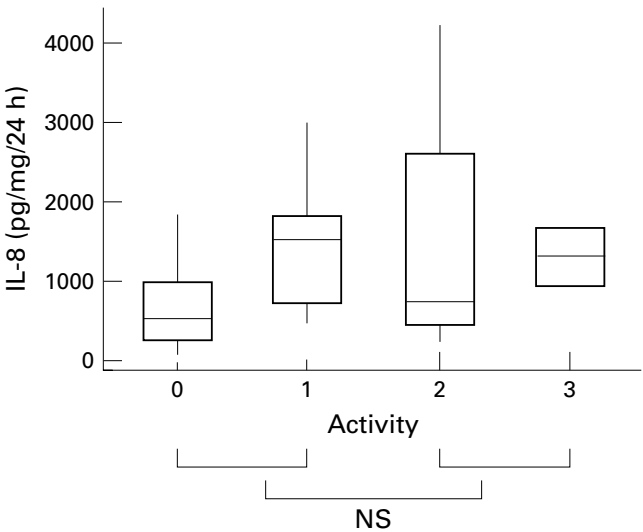

Figure 1 Concentration of tumour necrosis factor a (TNF-a) in 24 hour culture supernatants of "control" biopsies versus (A) antral inflammation (Sydney) score and (B) antral activity (Sydney) score. Concentration of interleukin 6 (IL-6) in 24 hour culture supernatants of "control" biopsies versus (C) antral inflammation (Sydney) score and (D) antral activity (Sydney) score. Concentration of interleukin 8 (IL-8) in 24 hour culture supernatants of "control" biopsies versus (E) antral inflammation (Sydney) score and $(F)$ antral activity (Sydney) score.

group were compared using the Wilcoxon matched pairs test. Immunohistochemistry scores for $H$ pylori positive and negative patients were compared using pooled score values by Fisher's exact test. All analyses used statistical software (Minitab v11.2, 1996; Minitab Ltd, Coventry, UK) and $\mathrm{p}$ values of $\leqslant 0.05$ were regarded as significant.

\section{Results}

CYTOKINE SECRETION

Biopsies from 41 patients, of whom 31 were $H$ pylori positive and 10 were negative on the basis of histology and biopsy urease testing of antral biopsies, were subject to in vitro culture.
Table 1 summarises the demographic and histological characteristics of the patients. The presence of the CagA protein was determined by western blotting in $18 \mathrm{H}$ pylori positive patients, of whom 13 were CagA seropositive and five were CagA seronegative.

Table 1 and figs 1 and 2 summarise the in vitro cytokine secretion. Considering control biopsies alone, the proinflammatory cytokine content of culture supernatants (TNF- $\alpha$, IL-6, and IL-8) was significantly greater in $\mathrm{H}$ pylori positive patients than in uninfected patients (table 1). The secretion of both TNF- $\alpha$ and IL-6 correlated both with the degree of chronic inflammation and with neutrophil activity, with 


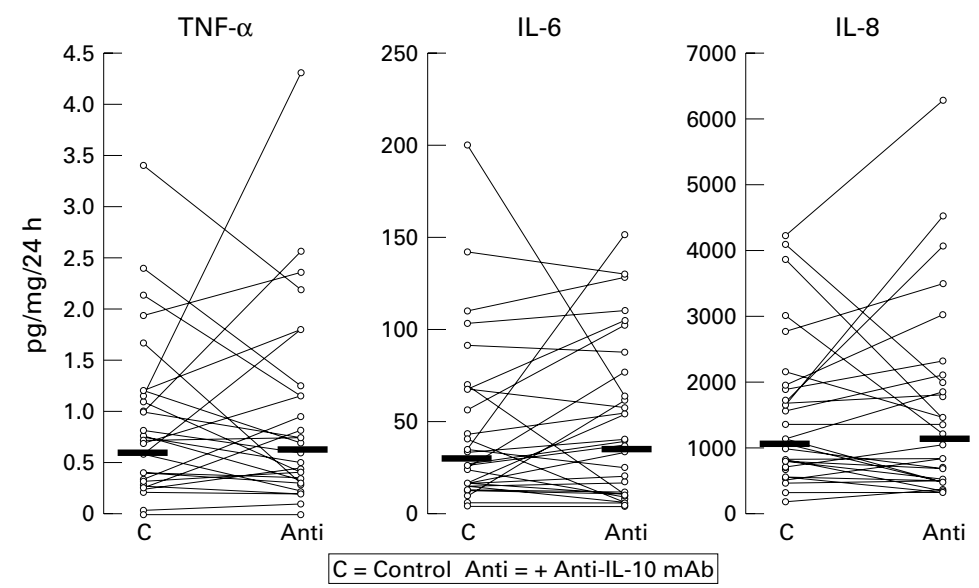

Figure 2 In vitro cytokine secretion for Helicobacter pylori positive patients: control biopsies versus biopsies cocultured in the presence of anti-IL-10 monoclonal antibody $(m A b)$. a significantly higher cytokine content in culture supernatants from patients having higher Sydney scores for these histopathological parameters (fig 1). There were no such trends for IL-8 secretion.

Within the subgroup of $H$ pylori positive patients in whom the CagA serological status was determined, the TNF- $\alpha$ content of culture supernatants was higher in CagA seropositive cases $(n=13)$ than in seronegative cases $(\mathrm{n}=5)$, with a median (IQR) secretion of 1.00

A

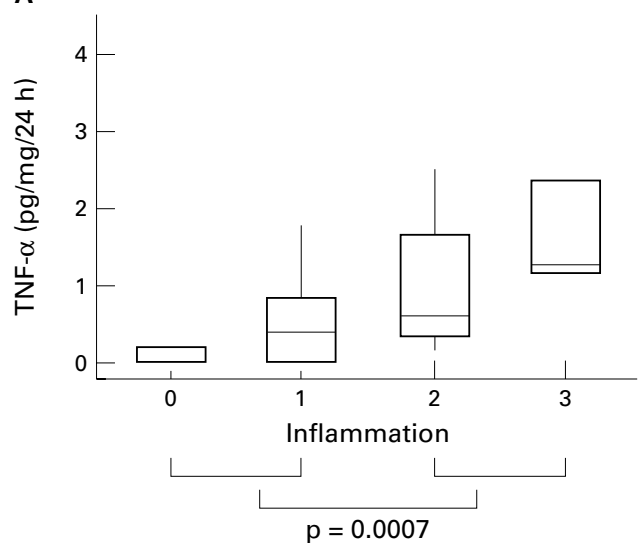

C

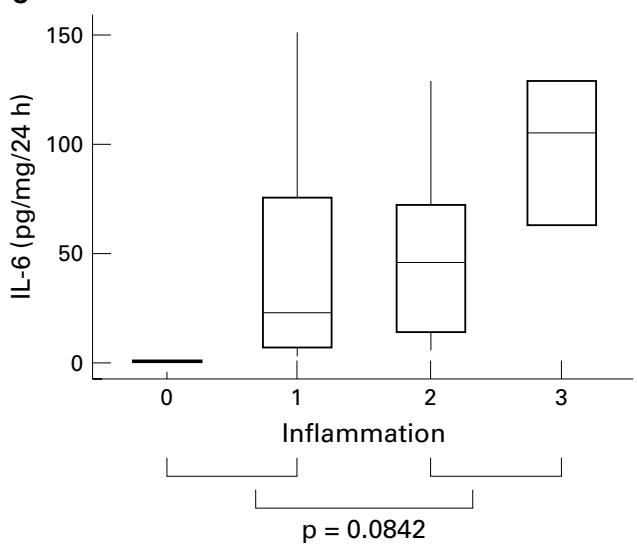

(0.295 to 1.435$)$ and 0.04 (0 to 0.705$)$ $\mathrm{pg} / \mathrm{mg} / 24$ hours, respectively $(\mathrm{p}=0.038$, Mann Whitney U test). IL-6 secretion was also higher in the CagA seropositive subgroup, with a median (IQR) secretion of 23.8 (15.3 to 54.0 ) versus 13.20 (6.95 to 26.20$) \mathrm{pg} / \mathrm{mg} / 24$ hours in the CagA seronegative group, although this did not reach significance $(p=0.11)$. There was no significant difference between the groups for IL-8 secretion, with median (IQR) values of 955 (717 to 1904) and 1650 (871 to 2252 ) pg/mg/24 hours, respectively ( $\mathrm{p}=0.55)$.

Figure 2 shows the effects of coculture with anti-IL-10 monoclonal antibody on cytokine secretion for the whole $H$ pylori positive group $(\mathrm{n}=31)$. Overall, there was no significant difference in proinflammatory cytokine secretion between control biopsies and those cocultured with neutralising anti-IL-10 monoclonal antibody. Stratifying the group by Sydney scores for the various histopathological parameters revealed no significant trends in the effects of coculture with anti-IL-10 monoclonal antibody. Indeed, secretion of $\mathrm{TNF}-\alpha$ and IL-6 in the presence of anti-IL-10 monoclonal antibody showed the same association with histopathology scores as the control biopsies (fig 3), but with no evidence of heightened cytokine secretion. Cytokine secretion in

B

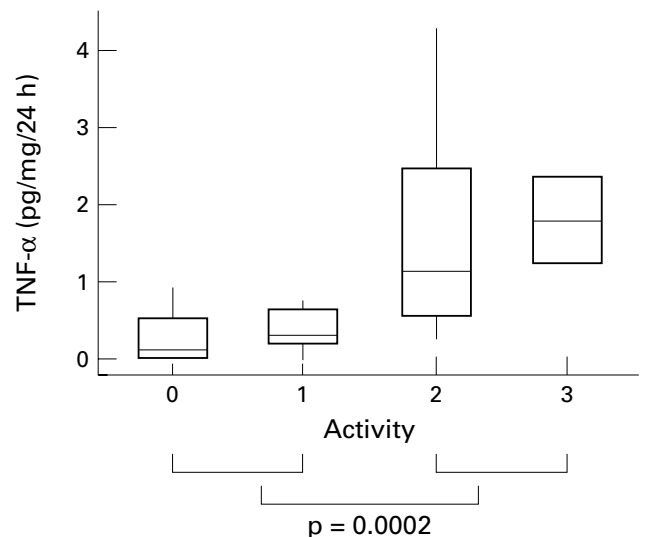

D

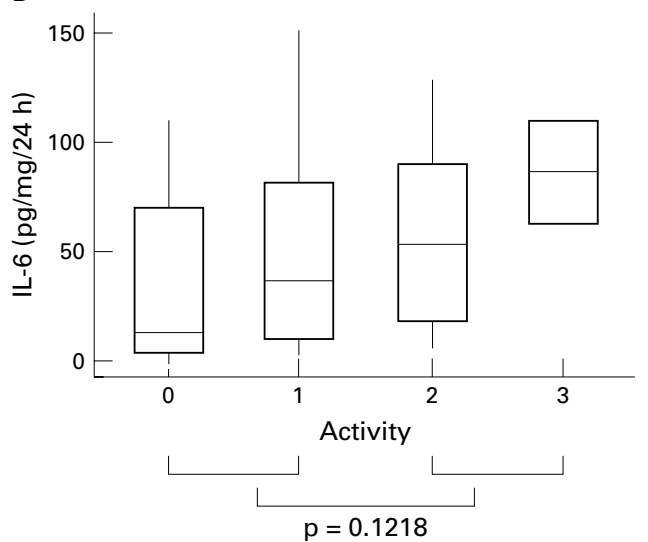

Figure 3 Concentration of tumour necrosis factor a (TNF-a) in 24 hour culture supernatants of biopsies cocultured in the presence of anti-IL-10 monoclonal antibody versus (A) antral inflammation (Sydney) score and (B) antral activity (Sydney) score. Concentration of interleukin 6 (IL-6) in 24 hour culture supernatants of biopsies cocultured in the presence of anti-IL-10 monoclonal antibody versus (C) antral inflammation (Sydney) score and (D) antral activity (Sydney) score. 

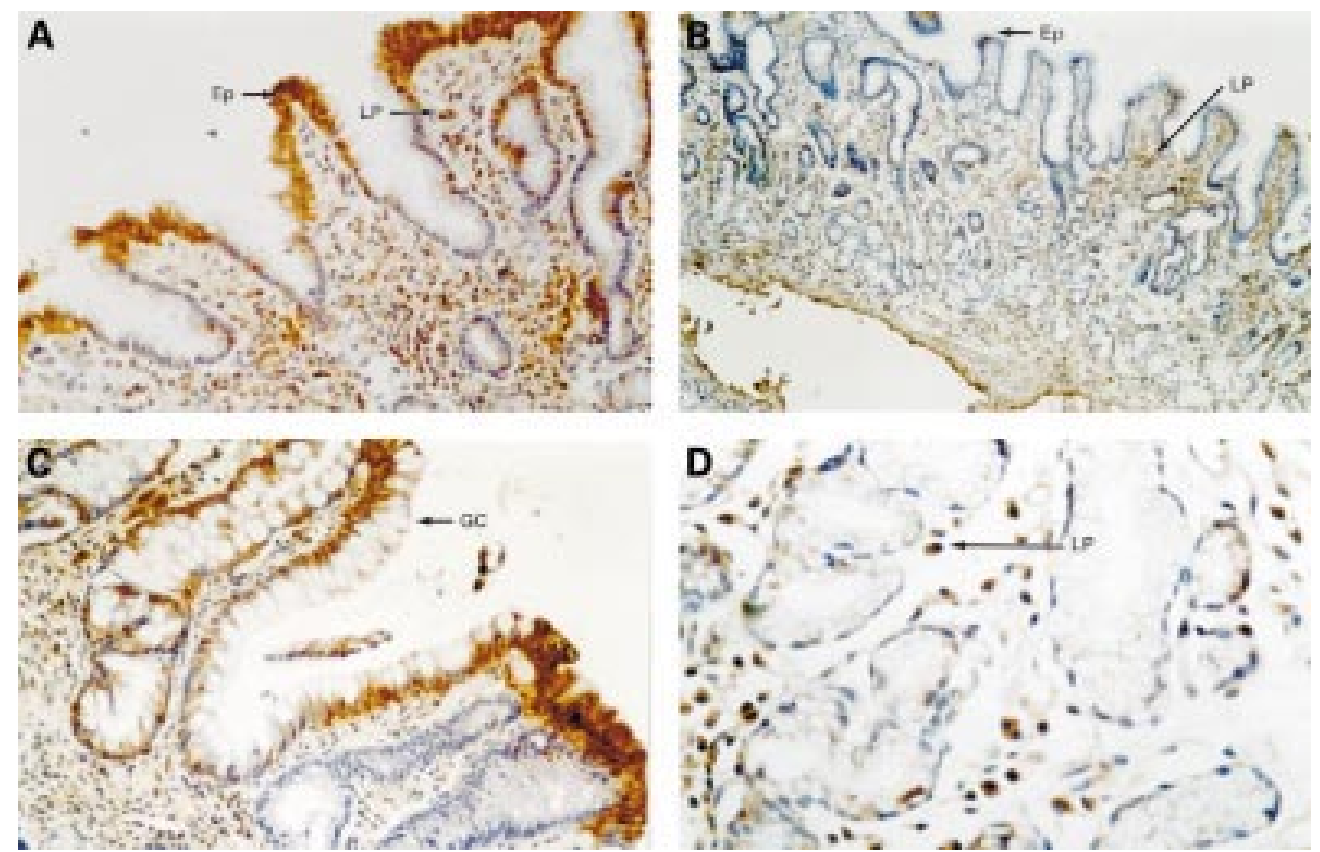

Figure 4 Immunohistochemical localisation of the interleukin 10 (IL-10) protein in formalin fixed gastric mucosal biopsies. (A) Helicobacter pylori positive patient: strong positivity in the surface epithelium (Ep) and frequent lamina propria mononuclear cells (LP). Original magnification, $\times 200$. (B) An H pylori negative patient: weak focal positivity in surface epithelium (EP) and scattered positive lamina propria (LP). Original magnification, $\times 100$. (C) A patient with $H$ pylori associated gastritis: strong cytoplasmic positivity of goblet cells (GC) in an area of intestinal metaplasia. Original magnification, $\times 200$. (D) A patient with $H$ pylori associated gastritis: high power view of lamina propria showing frequent positive lamina propria mononuclear cells (LP). Original magnification, $\times 400$.

the presence of anti-IL-10 monoclonal antibody did not differ in relation to CagA status.

IMMUNOHISTOCHEMISTRY

Immunostaining for IL-10 was performed on formalin fixed, paraffin wax embedded sections of antral biopsies from 29 patients, of whom 13 were $H$ pylori positive (chronic gastritis) and 16 were $H$ pylori negative. Control sections (no primary antiserum) were uniformly negative in all cases.

All sections from $H$ pylori positive patients and 13 of $16 \mathrm{H}$ pylori negative patients studied showed some epithelial cell positivity (fig 4A and B). Diffuse cytoplasmic immunoreactivity of surface epithelial cells was apparent, focally extending down into foveoli. Staining was most intense in areas where surface epithelial degeneration was most severe. Foveoli were largely

Table 2 Expression of interleukin 10 (IL-10) protein by gastric epithelium and lamina propria mononuclear cells (LPMNCs) in Helicobacter pylori positive (chronic gastritis) and H pylori negative (normal mucosa) patients

\begin{tabular}{lll}
\hline & $\begin{array}{l}\text { Helicobacter pylori } \\
+v e\end{array}$ & $\begin{array}{l}\text { Helicobacter pylori } \\
-v e\end{array}$ \\
\hline $\begin{array}{l}\text { No. of cases studied } \\
\text { Epithelium }\end{array}$ & 13 & 16 \\
Absent (0) & 0 & 3 \\
Weak (1) & 1 & 3 \\
Moderate (2) & 7 & 8 \\
Strong (3) & 5 & 2 \\
LPMNCs & & \\
Absent (0) & 0 & 4 \\
$<10 \%(1)$ & 1 & 5 \\
$10-50 \%(2)$ & 11 & 7 \\
$>50 \%(3)$ & 1 & 0 \\
\hline
\end{tabular}

^No significant difference between $H$ pylori +ve $v H$ pylori -ve. ${ }^{\star \star}$ Proportion of LPMNCs stained for IL-10 protein: $\mathrm{p}<0.05$ for $H$ pylori +ve $v$ H pylori -ve (Fisher's exact test) using pooled values (scores $0-1$ and $2-3$ ). spared and antral glands were generally negative, with occasional weak cytoplasmic positivity. In general, $H$ pylori positive cases had more extensive epithelial staining than $H$ pylori negative cases with normal mucosa, reflecting more widespread epithelial degeneration. Overall, the proportion of cases exhibiting absent/weak versus moderate/strong epithelial staining did not differ significantly with respect to $H$ pylori status (table 2 ).

In sections containing areas of intestinal metaplasia (fig 4C), foci of complete intestinal metaplasia were either negative or showed weak positivity in the surface but not in the crypts. Incomplete intestinal metaplasia showed diffuse cytoplasmic positivity of non-goblet cell surface epithelium throughout, with less strong positivity in glands.

In all $H$ pylori positive cases, some positive staining of lamina propria mononuclear cells (LPMNCs) was apparent (table 2; fig 4D), particularly in biopsies exhibiting more florid chronic inflammation. Positive cells were scattered diffusely in the lamina propria. Dense lymphoid aggregates were not stained. Positive LPMNCs were also seen in $H$ pylori negative cases, although the proportion of positive cells was less than in $H$ pylori positive cases. No immunostaining of neutrophils was seen.

\section{Discussion}

Our previous observation of IL-10 secretion from antral mucosa in $H$ pylori infection led us to speculate that endogenous secretion of IL-10 might damp down the immune response to infection, limiting tissue damage but also potentially contributing towards the failure of the immune response to clear the organism. ${ }^{3}$ In 
our present study, we aimed to demonstrate the in vitro effects of endogenously released IL-10 on the production of proinflammatory peptides in $H$ pylori infection and to determine possible sources of gastric IL-10 by means of immunohistochemistry.

Our present study confirms previous observations of enhanced proinflammatory cytokine release in $H$ pylori associated chronic gastritis, ${ }^{3-9}$ with significantly greater amounts of TNF- $\alpha$, IL- 6 , and IL-8 in culture supernatants (control biopsies) from $H$ pylori positive patients than from $H$ pylori negative patients. The secretion of both TNF- $\alpha$ and IL- 6 correlated with the degree of acute and chronic inflammation, reflecting enhanced cytokine production from more severely inflamed biopsies. We found enhanced production of $\mathrm{TNF}-\alpha$ and IL- 6 in a subgroup of $H$ pylori infected patients whose sera contained antibodies to the CagA protein (compared with CagA seronegative cases). This was not significant in the case of IL-6 and secretion of IL-8 was not higher in CagA seropositive cases. Molecular analysis of gastric mRNA has suggested that infection with cagA + strains is associated with increased transcription of IL- 8 , IL- $1 \alpha$, and IL- $1 \beta .^{1}{ }^{2} 89$

Coculturing antral biopsies in the presence of neutralising anti-IL-10 antibody did not produce the expected increase in TNF- $\alpha$, IL-6, and IL- 8 release. There are several possible explanations for this. First, there are clear methodological limitations to our in vitro approach. Although the amount of anti-IL-10 antibody added to the culture medium would have been sufficient to block anticipated endogenous IL-10 release, penetration of the neutralising antibody into the biopsies might have been limited. Coculture of human intestinal biopsies has been used to study the effects of therapeutic agents on the release of inflammatory mediators in vitro, ${ }^{14}{ }^{15}$ but not to our knowledge in studying endogenous cytokine actions. Second, cytokine pathways are highly complex with individual peptides exhibiting diverse and often overlapping functions. The effects of blocking IL-10 bioactivity in organ culture might have been masked by secondary changes in the secretion of other cytokines, serving to sustain proinflammatory peptide secretion. In view of these limitations, an important role for IL-10 in reducing gastric immune responses to $H$ pylori cannot be excluded. Recent observations of a rapidly progressive hyperplastic gastritis in IL-10 (-/-) knockout mice infected with a gastric helicobacter $^{12}$ argue in favour of our hypothesis that IL-10 does indeed play a key role in determining the severity of the local immune response in $H$ pylori associated gastritis.

There has been considerable recent interest in the possible role of IL-10 in the gut. The chronic intestinal inflammation seen in the IL-10 (-/-) knockout mouse suggests that, in health, the peptide might reduce local inflammation stimulated by normal enteric antigen exposure. ${ }^{11}$ Expression of IL-10 mRNA has been observed in normal colon tissues, ${ }^{18}$ although the cellular source was not clear. Isolated colonic lamina propria $\mathrm{T}$ cells have been reported to release high amounts of IL-10 on activation by a variety of stimuli, suggesting one potential cellular source. ${ }^{19}$ In a recent report, the in situ expression of IL-10 protein and mRNA was seen in both normal colon and in inflammatory bowel disease, ${ }^{20}$ with expression localised to epithelial cells as well as LPMNCs.

Despite evidence of IL-10 expression/ secretion by whole gastric biopsies, ${ }^{37}$ there are no reports of in situ localisation of the cellular sources of production. Although originally described as a product of T helper type 2 (Th2) lymphocytes, IL-10 is produced by a variety of cell types, notably mononuclear phagocytes. ${ }^{13}$ Present evidence suggests that a Th1 response predominates in $H$ pylori infection, ${ }^{21}$ such that the production of IL- 10 by Th2 cells is unlikely to be a major source in gastritis.

In our present study, we localised IL-10 protein in formalin fixed, paraffin wax embedded gastric tissues using a combination of microwave antigen retrieval and standard peroxidase immunohistochemistry. Because of the absence of recognised control tissues for IL-10 immunostaining, we cannot be completely confident of the specificity of staining for IL-10. However, Autschbach et al have reported a close correlation between immunostaining of colonic tissue using the same primary antibody (MAB 217; R\&D Systems) and the in situ expression of IL-10 mRNA, suggesting that immunostaining with this antibody provides accurate localisation of IL-10. ${ }^{20}$

We observed epithelial cell immunostaining for IL-10 protein in almost all sections, including both $H$ pylori associated gastritis and normal mucosa. Although the strongest epithelial reaction was seen in $H$ pylori positive cases in areas of epithelial degeneration, overall there was no significant difference in epithelial reactivity between inflamed biopsies ( $H$ pylori positive) and normal mucosa.

These findings are similar to previous observations of epithelial IL-10 protein and mRNA expression in both inflamed and non-inflamed colon. ${ }^{20}$ Similarly, the proinflammatory chemokine, IL-8, can be demonstrated in gastric epithelial cells by means of immunohistochemistry in both normal and inflamed gastric tissues, ${ }^{122}$ suggesting constitutive expression of immunoregulatory peptides in human gastric epithelium. Epithelial IL-10 secretion could reduce local immune responses against luminal antigens, contributing to a state of "controlled inflammation" in the normal intestine, ${ }^{23}$ and influencing the clinicopathological outcome of mucosal infection.

\section{Conclusion}

IL-10 is a potent counterinflammatory peptide implicated in the downregulation of human intestinal immune responses. Our study localised IL-10 protein in situ to the gastric epithelium and LPMNCs in both normal mucosa and $H$ pylori gastritis, a phenomenon not reported previously. Although blocking IL-10 bioactivity in organ culture did not produce enhanced proinflammatory peptide release from the antral mucosa, further studies of the 
role of IL-10 in $H$ pylori infection are required. In agreement with previous studies, we have shown that proinflammatory peptides such as TNF- $\alpha$, IL-6, and IL- 8 are secreted in $H$ pylori gastritis, and our data demonstrate that the secretion of TNF- $\alpha$ and IL- 6 correlate with increased severity of acute and chronic inflammation, and that concentrations of these molecules are increased in patients infected with cagA positive strains in particular.

1 Bodger K, Crabtree JE. H. pylori and inflammation. $\mathrm{Br} \mathrm{Med}$ Bull 1998;54:139-50.

2 Atherton J. H. pylori virulence factors. $\mathrm{Br}$ Med Bull 1998;54:105-20.

3 Bodger K, Wyatt JI, Heatley RV. Gastric mucosal secretion of interleukin-10: relations to histopathology, $\mathrm{H}$. pylori status and TNF- $\alpha$ secretion. Gut 1997;40:739-44.

4 Crabtree JE, Shallcross TM, Heatley RV, et al. Mucosal Crabtree JE, Shallcross TM, Heatley RV, et al. Mucosal with Helicobacter pylori-associated gastritis. Gut 1991;44: with $\mathrm{He}$

5 Crabtree JE, Wyatt JI, Trejdosiewicz LK, et al. Interleukin-8 expression in Helicobacter pylori, normal and neoplastic gastroduodenal mucosa. F Clin Pathol 1994;47:61-6.

6 Noach LA, Bosma NB, Jansen J, et al. Mucosal tumour necrosis factor-alpha, interleukin-1 beta, and interleukin-8 production in patients with Helicobacter pylori infection. Scand $\mathcal{F}$ Gastroenterol 1994;29:425-9.

7 Yamaoka Y, Kita M, Kodama T, et al. Expression of cytokine mRNA in gastric mucosa with Helicobacter pylori infection. Scand 7 Gastroenterol 1995;30:1153-9.

8 Peek RM, Jr, Miller GG, Tham KT, et al. Heightened inflammatory response and cytokine expression in vivo to CagA + Helicobacter pylori strains. Lab Invest $1995 \cdot 73$ : 760-70.

9 Yamaoka Y, Kita M, Kodama T, et al. Helicobacter pylori cagA gene and expression of cytokine messenger RNA in gastric mucosa Gastroenterology 1996;110:1744-52.

10 Fiorentino DF, Zlotnic A, Mossmann TR, et al. IL-10 inhibits cytokine production by activated macrophages. $\mathcal{f}$ inhibits cytokine production by activated macrophages. $\mathcal{F}$

11 Kuhn R, Lohler J, Rennick D, et al. Interleukin-10-deficient mice develop chronic enterocolitis. Cell 1993;75:263-74.
12 Berg JB, Lynch NA, Lynch RG, et al. Rapid development of severe hyperplastic gastritis with epithelial dedifferentiation in Helicobacter felis-infected IL-10 -/- mice. Am F Pathol 1998;152:1377-86.

13 Dixon MF, Genta RM, Yardley JH, et al. International workshop on the histopathology of gastritis, Houston 1994: classification and grading of gastritis: the updated Sydney system. Am 7 Surg Pathol 1996;20:1161-81.

14 Wardle TD, Hall L, Turnberg LA. Use of co-culture of colonic mucosal biopsies to investigate the release of eicosanoids by inflamed and uninflamed mucosa from patients with inflammatory bowel disease. Gut 1992;33: 1644-51.

15 Reimund JM, Dumont S, Muller CD, et al. In vitro effects of oxpentifylline on inflammatory cytokine release in patients with inflammatory bowel disease. Gut 1997;40:475-80

16 Shi S-R, Key ME, Karla KL. Antigen retrieval in formalinfixed, paraffin-embedded tissues: an enhanced method for immunohistochemical staining based on microwave heating of tissue sections. F Histochem Cytochem 1991;39:741-8.

17 Terada T, Kitamura Y. Immunocytochemical investigation of paraffin-embedded tissue. In: Preedy PR, Watson RR, eds. Methods in disease: investigating the gastrointestinal tract. London: Greenwich Medical Media Ltd, 1998:301-9.

18 Niessner M, Volk BA. Altered Th1/Th2 cytokine profiles in the intestinal mucosa of patients with inflammatory bowel disease as assessed by quantitative reverse transcribed polymerase chain reaction (RT-PCR). Clin Exp Immunol 1995;101:428-35.

19 Braunstein J, Qiao L, Autschbach F, et al. T cells of the human intestinal lamina propria are high producers of IL-10. Gut 1997;41:215-20.

20 Autschbach F, Braunstein J, Helmke B, et al. In situ expression of interleukin-10 in non-inflamed human gut and in inflammatory bowel disease. Am F Pathol 1998;153:12130 .

21 D'Elios MM, Manghetti M, De Carli M, et al. T helper 1 effector cells specific for Helicobacter pylori in the gastric antrum of patients with peptic ulcer disease. F Immunol 1997;158:962-7.

22 Crabtree JE, Wyatt JI, Trejdosiewicz LK, et al. Interleukin-8 expression in Helicobacter pylori gastritis, normal and neoplastic gastroduodenal mucosa. $\mathcal{F}$ Clin Pathol 1994;47: $61-6$

23 O'Farelly C. Just how inflamed is the normal gut? Gut 1998; 42:603-6

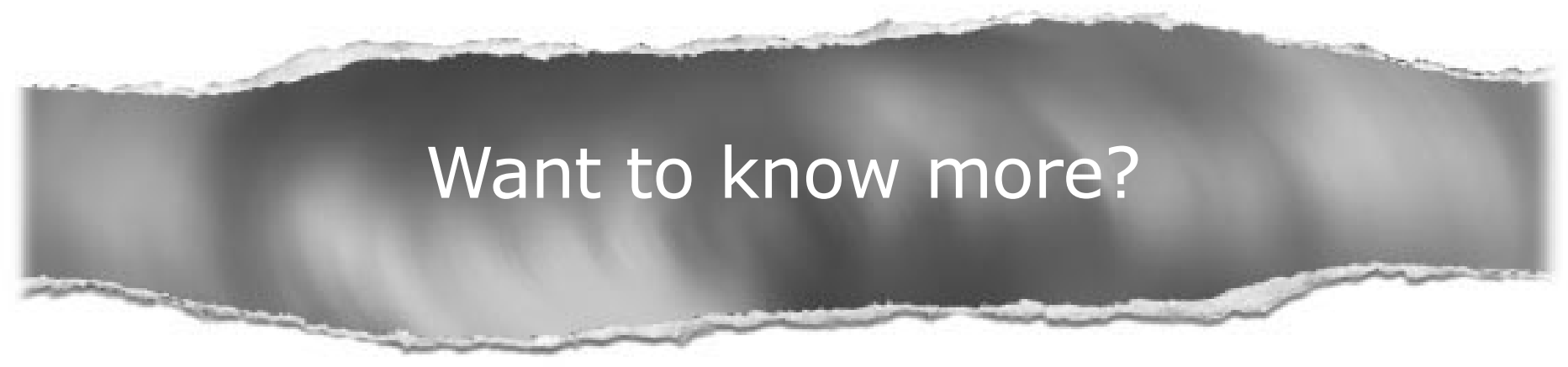

Data supplements

Limited space in printed journals means that interesting data and other material are often edited out of articles; however, limitless cyberspace means that we can include this information online.

Look out for additional tables, references, illustrations.

www.jclinpath.com 